\title{
Displacement of all three leaflets of tricuspid valve: a rare variant of Ebstein anomaly
}

\author{
Zahra Khalaji ${ }^{1}$, Nahid Rezaeian ${ }^{1}$, and Zahra Ansari ${ }^{1}$ \\ ${ }^{1}$ Iran University of Medical Sciences
}

March 8, 2021

\begin{abstract}
Ebstein anomaly, a rare congenital heart disease, is defined as displacement of hinge points of septal or posterior tricuspid leaflets but not anterior leaflet. Here we report a 35 year old lady with an extremely rare pattern of EA with all three tricuspid leaflets displaced downward to the apex.
\end{abstract}

\section{INTRODUCTION}

Ebstein anomaly is a rare form of congenital heart disease with approximately one in 20000 live births ${ }^{1}$. EA characterized by septal and posterior leaflets downward displacement into the body of right ventricle with the anterior leaflet remaining in the normal position ${ }^{2,3,4}$. Here we present a rare case of EA with apical displacement of all 3 leaflets of tricuspid valve.

\section{PRESENTATION OF CASE}

A 35 year-old lady was referred to adult congenital heart disease department due to recently presented dyspnea on exertion and systolic murmur. Transthoracic echocardiography indicated an enlarged RA with apical displacement of septal $(1.6 \mathrm{~cm} / \mathrm{m} 2)$, posterior $(1.5 \mathrm{~cm} / \mathrm{m} 2)$ and also anterior $(1.2 \mathrm{~cm} / \mathrm{m} 2)$ tricuspid leaflets (figure 1). Severe right atrium enlargement and severe low gradient tricuspid regurgitation (TRG=26 $\mathrm{mmhg}$ ) was also recorded. Interatrial septum was aneurysmal with large PFO. Left ventricular function was mildly reduced with hypertrabeculation and apical non-compaction. Chest X ray showed increased cardiothoracic ratio with enlarged RA size (figure 2). Electrocardiogram showed first degree AV block, Q in leads III and aVF and narrow QRS (figure 3). Cardiac magnetic resonance (CMR) was done to evaluate cardiac function, anatomy as well as fibrosis. It was demonstrated an extraordinary case of Ebstein anomaly with apical displacement of all anterior $\left(35 \mathrm{~mm} / \mathrm{m}^{2}\right.$ BSA $)$, septal $\left(18 \mathrm{~mm} / \mathrm{m}^{2} \mathrm{BSA}\right)$ and posterior $\left(20 \mathrm{~mm} / \mathrm{m}^{2}\right.$ BSA) tricuspid valve leaflets (figure 4). Anterior trictspid valve leaflet was short and had some fenestration with severe TR. CMR also mentioned a prominent ridge in RA at the site of AV groove, which protruded to RV cavity with attachment of anterior TV leaflet to it.

\section{DISCUSSION}

Ebstine anomaly is essentially a tricuspid valve disorder which begins in the embryonic stage by failure of delamination ${ }^{2,5}$. Due to failure in delamination of inner layer of inlet part of right ventricle in EA we see adherence of septal and posterior leaflets to the underlying myocardium and as a consequence we saw downward displacement of hinge points of septal and posterior leaflets from atrioventricular junction ${ }^{2,5,6}$. In contrast to septal and posterior leaflets, anterior leaflet usually remains in the normal position due to different embryonic origin of anterior leaflet versus posterior and septal leaflets ${ }^{4,7}$. Septal (medial) and posterior (inferior) leaflets originate from the tricuspid gully ${ }^{7}$ but the anterior leaflet developed from the lateral endocardial cushion and lateral conus ${ }^{2}$, so that, anterior tricuspid leaflet is very rarely affected in EA 
and the attach point of the septal and inferior leaflets never apically displaced beyond the junction between ventricular inlet and apicotrabecular component of $\mathrm{RV}^{1,7}$ and the junctional hing of the anterior leaflet is very rarely affected ${ }^{2,7}$.

In our case which the symptoms represented in adulthood, a rare variant of EA was seen with downward displacement of all three tricuspid leaflets. Ebstein cases may represent at different ages due to severity of structural and functional abnormality and also presence of associated defects ${ }^{8}$, such as our patient which well tolerated till 35 years old and passed tow pregnancies with no complain of dyspnea or right side failure symptoms. There was no same case in literature with apical disposition of all three tricuspid leaflets. There was reported few cases of isolated anterior leaflet displacement and not septal and posterior ones, and displacement of septal or posterior leaflets is the regular kind of EA, but apical downward of all three ones was not reported till now.

\section{CLINICAL KEY MASSEGE}

There are some reports of few cases of isolated anterior leaflet displacement without septal and posterior leaflets but apical downward of all three tricuspid leaflets as we saw in our present case was not reported till now.

\section{CONFLICT OF INTEREST}

The authors have no conflicts of interest to declare.

\section{AUTHOR CONTRIBUTION \\ REFERENCES}

1. Mulder, B.J.M. 2003. Ebstein's anomaly in the adult patient. Netherlands Heart Journal , 11 (5): 195-198.

2. Tsujii, N., Kurosaki, K., Yasuda, K., Mizuno, M., Sakaguchi, H., Hoashi, T., Ichikawa, H. and Shiraishi, I. 2016. Displacement of the anterior leaflet of the tricuspid valve: Rare variant of Ebstein's anomaly. Pediatrics International , 58 (8): 775-777.

3. Luu, Q., Choudhary, P., Jackson, D., Canniffe, C., McGuire, M., Chard, R. and Celermajer, D.S., 2015. Ebstein's anomaly in those surviving to adult life-a single centre experience. Heart, Lung and Circulation , 24 (10), pp.996-1001.

4. Wu, Q., Pan, G., Li, H. and Kong, X. 2014. Anatomic repair of Ebstein's anomaly with isolated anterior leaflet downward displacement. The Journal of thoracic and cardiovascular surgery , 148 (4):1454-1458.

5. Attenhofer Jost, C.H., Connolly, H.M., Dearani, J.A., Edwards, W.D. and Danielson, G.K., 2007. Ebstein's anomaly. Circulation, 115 (2), pp.277-285.

6. Muñoz-Castellanos, L., Espinola-Zavaleta, N., Kuri-Nivón, M. and Keirns, C. 2007. Ebstein's Anomaly: Anatomo-echocardiographic correlation. Cardiovascular Ultrasound , 5 (1): 1-10.

7. Lamers, W.H., Viragh, S., Wessels, A., Moorman, A.F. and Anderson, R.H. 1995. Formation of the tricuspid valve in the human heart. Circulation, 91 (1): 111-121.

8. Tabatabaei, N., Katanyuwong, P., Breen, J.F., Glockner, J., Dearani, J.A., Khouzam, R.N. and Ammash, N.M. 2009. Uncommon variant of Ebstein anomaly with tricuspid stenosis. Circulation, 120 (1): e1-e2.

Figures legends:

Figure 1. Transthoracic echocardiography. A: four chamber view showed apical displacement of all three tricuspid valve leaflets with no anterior leaflet elongation. B: Parasternal short axis view showed apical non compaction of left ventricle as an associated anomaly. 
Figure 2. Anteroposterior chest $\mathrm{X}$ ray. Note increased cardiothoracic ratio due to enlarged right ventricle and also right atrium due to Ebstein anomaly and severe tricuspid regurgitation.

Figure 3. Electrocardiogram showed narrow QRS with first degree AV block. Note Q wave in II and aVF

Figure 4. CMR image demonstrates apical displacement of all tricuspid valve leaflets. A, B, C, and D show anterior tricuspid valve (yellow arrows), septal tricuspid (green arrow), and posterior tricuspid valve (thick pink arrows) displacement. The Redline depicts the approximate level of the mitral valve. E. Two-chamber cine function shows non-compaction LV

\section{Hosted file}

Figures.pdf available at https://authorea.com/users/318924/articles/512533-displacement-ofall-three-leaflets-of-tricuspid-valve-a-rare-variant-of-ebstein-anomaly 